\title{
RELIABILITY ESTIMATION FOR COMPONENTS OF PHOTOVOLTAIC SYSTEMS
}

\author{
P. R. MISHRA ${ }^{1}$ and J. C. JOSHI ${ }^{2 *}$ \\ ${ }^{1}$ Central Electronics Ltd, Sahibabad 201010 , India \\ ${ }^{2}$ Centre for Energy Studies, Indian Institute of Technology, Hauz Khas, New Delhi 110016, India
}

(Received 11 January 1995; received for publication 20 September 1995)

\begin{abstract}
In this study, a reliability estimation method was used to predict the probability of success of various components of photovoltaic (PV) systems. Various methods of reliability improvement, to improve the probability of success of the PV system for the least annualised cost, are discussed. This paper mathematically estimates and analyses the reliability estimation of the battery voltage regulators (BVRs) that are generally used in PV systems. Therefore, this can be of vital help to a PV system designer for improving the reliability of the BVRs. The paper also describes different reliability engineering techniques for improving the probability of success of PV system components. In the case study, those BVRs whose probability of success is $18 \%$ in the laboratory condition, were actually found to have less than $1 \%$ probability of success if subjected to tropical field conditions for the expected life period of $10 \mathrm{yr}$. For the same BVR, the probability of success increases to $99 \%$ for the same period if proper reliability-related design considerations are incorporated.
\end{abstract}

Reliability Probability of success Derating Redundancy

\section{INTRODUCTION}

Reliability engineering is very important in areas of engineering products. According to British Standards, reliability is defined as "the ability of an item to perform a required function under stated conditions for a stated period of time". NASA defines reliability as "the probability of a device performing adequately for the period of time under the operating conditions encountered". This is more relevant to photovoltaic (PV) systems because they have long pay-back periods, remote installations and new technology. The PV industry is also entering into large-scale commercial deployment from the pilot demonstration project level.

Reliability, in other words, is the successful operation of the systems. The success of PV systems will be measured by the availability of the power source to the load during its expected life time. Thus, the availability (A) is the probability of functioning at any specific time in the expected life time:

$$
\mathrm{A}=\text { operable time } / \text { mission time }=\mathrm{MTBF} / \mathrm{MTBF}+\mathrm{MTTR}
$$

where MTBF is the mean time between failures and MTTR is the mean time to repair. In a PV system, the MTTR is very high due to the non-availability of skilled manpower, spares and proper tools near the power plant. Thus, failures in PV systems will result in loss of service for long periods. This will increase the pay-back period. In such situations, the MTBF, especially the mean time to first failure (MTTF), becomes almost equal to the life of the equipment; thus, it is very important that proper design considerations enhance the MTBF, i.e. the life of the equipment, and will result in a shorter pay-back period. In addition, it is a fact that the characteristics of a PV plant are different from those of the conventional power plant. As such, it requires compatible and adoptable interfacing equipment, e.g. low maintenance and deep cycle life battery, battery voltage regulator (BVR), high efficiency inverters, etc. Failure rates of this equipment are largely unknown. 
Therefore, it is very important to go into minute details of reliability and predict the reliability estimation of this equipment before launching the products for commercial deployment.

The PV system, in general, contains PV panels, batteries, a BVR, MPPT (maximum power point tracker) and load. The reliability of the PV system will depend upon the reliability of its constituent equipment. The pay-back period of a PV system is approximately $10 \mathrm{yr}$, so the reliability estimation should be done at least for $10 \mathrm{yr}$ or more.

In this study, different reliability techniques were undertaken to examine two different types of BVR, the conventional and the new BVR. The calculation has been done using different reliability techniques to increase the life of the equipment to an optimum level. This study can be further extended to other parts of the PV system.

\section{THEORY}

Reliability can be defined in quantitative terms as the probability that the device will meet the qualitative definition. If $T$ is the time to failure of the device ( $T$ itself is a variable), then the probability that it will not fail in the given environment before time $t$, i.e. its reliability is given as [1]

$$
R(t)=P(T>t)
$$

The device is assumed to be working properly at time $t=0$ and cannot work forever $(t=\infty)$ without failures. These statements are expressed as

$$
R(0)=1 \quad R(\infty)=0
$$

The mathematical definition of reliability engineering generates three tasks. The first task is to derive and find out the physical interpretation of equation (1). This is done for a single component. Then, the reliability of an entire system is determined considering the configuration and functions of the components taking part in the system. The building process continues until the complete system under consideration has been treated. The second task is to find the best way of increasing the reliability. The third task is to maximise systems reliability for a given weight, size or cost.

In order to derive the reliability equation, the interrelation of reliability $R(t)$ and failure rate of the system can be given as

$$
f(t)=f^{\prime}(t) / R(t)
$$

where $f^{\prime}(t)$ is the failure density function and $f(t)$ is defined as the instantaneous failure rate. It can be given as

$$
f(t)=-[1 / R(t)] *\{\mathrm{~d} R(t) / \mathrm{d} t\}
$$

where $\mathrm{d} R \backslash \mathrm{d} t$ represents the slope of the reliability function. Integrating equation (4) from time 0 to time $t$

$$
R(t)=\exp \left[-\int_{0}^{t} f(t) \mathrm{d} t\right]
$$

In the case of a constant failure rate, i.e. $f(t)$ is independent of time, is of special interest, then

$$
-\int_{0}^{t} f(t) \mathrm{d} t=-f t
$$

The special case of the useful life with constant failure rate is given as

$$
R(t) \mathrm{d} t=\exp (-f t)
$$

and the MTBF, especially the MTTF, can be written as

$$
\text { MTTF }=\exp (-f t) \mathrm{d} t=1 \bigvee f
$$


The reliability of a component can be obtained from equation (7). In the real world, any system consists of more than one component. They may be connected in series, in parallel or some combination of series and parallel.

A series system is one in which all components are so interrelated that the entire system will fail if any of its components fails, whereas a parallel system is one which will fail only if all its components fail.

Considering $n$ components connected in series, the reliability of system can be calculated as

$$
\begin{aligned}
R \text { system } & =R_{1} \times R_{2} \times \ldots R_{n} \\
& =\exp \left[-\left(f_{1} t\right)\right] \times \exp \left[-\left(f_{2} t\right)\right] \ldots \exp \left[-\left(f_{n} t\right)\right] \\
& =\exp -\left\{\left(f_{1}+f_{2}+\ldots f_{n}\right) t\right\} \\
f(\text { system }) & =f_{1}+f_{2}+\ldots f_{n} \\
R(\text { system }) & =\exp -\{f(\text { system }) t\}
\end{aligned}
$$

From equation (11), it is clear that the failure rate of a system is the arithmetic summation of the fault rates of the components. For the $n$ components connected in parallel, the reliability of the system is calculated from the unreliability of the system:

$$
R(\text { system })=1-Q(\text { system })
$$

The unreliability of the system is

$$
Q(\text { system })=Q_{1} \times Q_{2} \times \ldots Q_{n}
$$

From equation (13),

$$
\begin{gathered}
R(\text { system })=1-Q_{1} \times Q_{2} \times \ldots Q_{n} \\
=1-\left(1-\exp \left(f_{1} t\right)\right)\left(1-\exp \left(f_{2} t\right)\right) \ldots\left(\exp \left(f_{n} t\right)\right)
\end{gathered}
$$

If $f_{1}=f_{2}=\ldots f_{n}=f$, then equation (16) will be simplified and equation (15) is given as

$$
R(\text { system })=1-\{1-\exp (f t)\}
$$

The failure rate of the system will be given as

$$
1 \bigvee(\text { system })=1 \bigvee f+1 \backslash 2 f+\ldots 1 \backslash n f
$$

\section{Effect of temperature and humidity on reliability}

Most of the failure mechanisms involve one or more physical or chemical processes, each occurring at a rate which is highly dependent on temperature. To predict the effect of temperature on failure rate, the most widely accepted model in reliability work is the Arrhenius model, which predicts an exponential increase in the rate of a given reaction with temperature [2]. The form of the model generally used in reliability is

$$
f=f_{0} \exp -\left\{E a \backslash K\left(1 \backslash T-1 \backslash T_{0}\right)\right\}
$$

where $f$ is the temperature-related failure rate, $f_{0}$ is a normalising rate constant or base failure rate, $E a$ is the activation energy $(\mathrm{eV}), K$ is Boltzmann's constant, $T$ is the ambient temperature $(\mathrm{K})$ and $T_{0}$ is the reference temperature $(\mathrm{K})$.

Similarly, the failure rate increases with an increase in humidity. The relation is given by [3]

$$
f_{1}=f_{0} \times\left(R H_{1} \backslash R H_{0}\right)^{n}
$$

where $R H_{1}$ is the relative humidity, $R H_{0}$ is the reference relative humidity, $n=2.7$ for our application, $f_{0}$ is the base failure rate and $f_{1}$ is the failure rate at humidity $R H_{1}$. 


\section{RELIABILITY ANALYSIS OF ELEMENTS/COMPONENTS OF THE PV POWER SOURCE}

The reliability of a PV power source will depend upon the reliability of its constituent components. The PV power source consists of the solar photovoltaic array (PA), battery bank (BB) and electronic control modules (ECM).

Since the performance of the power source is affected if any of the components malfunctions, the components were connected in series for reliability calculations, which is given as

$$
R(t)[\mathrm{PV} \text { system }]=R(t)_{\mathrm{PA}} \times R(t)_{\mathrm{BB}} \times R(t)_{\mathrm{ECM}}
$$

Since the $R(t)$ of the PA is independent of the $R(t)$ of both the BB and the ECM, for the present case, we restricted ourselves to the study of the rest of the system. From here, the $R(t)$ [system] will mean the reliability of the rest of the system, except for the PA. Therefore,

$$
R(t)[\text { system }]=R(t)_{\mathrm{BB}} \times R(t)_{\mathrm{ECM}}
$$

The $R(t)$ of the BB will depend upon the depth of discharge (DOD) and the time interval between two consecutive discharges. The $R(t)$ of the ECM will depend upon the reliability of the different blocks used in the ECM.

For ease of analysis in our study, the ECM simply contained a battery voltage regulator (BVR). Since the $R(t)$ of the BB is dependent upon the type of BVR, two different types of BVR are discussed and compared. The block diagrams of the conventional BVR and the new BVR are given in Fig 1(a) and (b), respectively. The conventional BVR, in general, contains electromechanical

(a)

\begin{tabular}{|l|l|}
\hline \begin{tabular}{l|l|} 
Battery status \\
indications
\end{tabular} & $\begin{array}{l}\text { Protection against reverse } \\
\text { flow of current from } \\
\text { battery bank to array }\end{array}$ \\
\hline $\begin{array}{l}\text { Overcharge protection } \\
\text { circuit }\end{array}$ & $\begin{array}{l}\text { Charging current } \\
\text { cut-off switch }\end{array}$ \\
\hline $\begin{array}{l}\text { Low battery charge } \\
\text { protection circuit }\end{array}$ & Load cut-off switch \\
\hline Short circuit protection
\end{tabular}

(b)

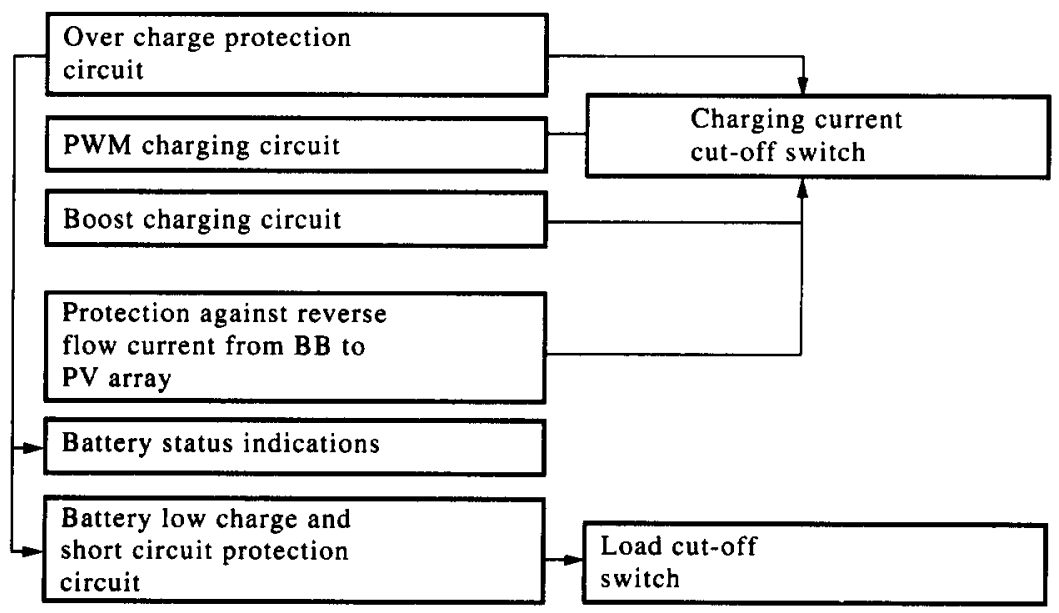

Fig. 1(a). Block diagram of conventional BVR, (b) block diagram of new BVR. 
Table 1. Calculation of failure rate of conventional BVR based on standard reliability level electronic parts

\begin{tabular}{llrrr} 
& & & \multicolumn{2}{c}{$\begin{array}{c}\text { Failure rate } \\
\left(\text { per } 10^{9} \mathrm{~h}\right)\end{array}$} \\
\cline { 4 - 6 } S. No. & $\quad$ Components & $\begin{array}{c}\text { Quantity/unit } \\
(n)\end{array}$ & $(f)$ & $n \times f$ \\
\hline 01. & Resistance (fixed) & 25 & 58 & 1450 \\
02. & Wire wound resistance & 01 & 174 & 174 \\
03. & Variable resistances & 04 & 122 & 488 \\
04. & Integrated circuits & 01 & 168 & 168 \\
05. & Transistor & 08 & 250 & 2000 \\
06. & Power diode & 01 & 378 & 378 \\
07. & Diode & 17 & 84 & 1428 \\
08. & Electrolytic capacitor & 09 & 429 & 3861 \\
09. & Ceramic capacitor & 05 & 214 & 1070 \\
10. & Connector pin & 08 & 40 & 320 \\
11. & Relay & 02 & 400 & 800 \\
12. & Circuit breaker & 01 & 220 & 220 \\
13. & Crimping & 20 & 11 & 220 \\
14. & Solder points (mech.) & 170 & 04 & 680 \\
15. & Solder points (hand) & 27 & 100 & 2700 \\
& & & & \\
\hline
\end{tabular}

relays, a circuit breaker and blocking diode besides other components, namely zener diode, diode, resistance, op-amb, etc.

The new BVR offers series regulation with minimal offset voltage in the charging circuit, an electronic fuse for accurate overload and short-circuit protection, pulse width modulated charging regulation and an automatic boost charging facility once a month, in contrast to the conventional BVR, which provides only on-off switching for charging regulation, one-volt drop over blocking diode and needs a circuit breaker for short-circuit protection.

We are now analysing the systems for the two BVRs on the basis of the following assumptions:

(1) all the components are assumed to be connected in series;

(2) the current ability to the load is higher by $10 \%$ when the new BVR is connected in the system in place of the conventional BVR [4]; and

(3) no-sunshine days follow full-sunshine days alternately.

To calculate the reliability and life of the battery bank, it has been described already that the DOD and time interval between consecutive deep discharges should be known.

From assumption (2), it can be calculated that a battery which was allowed to discharge up to $40 \%$ DOD, for a certain system configuration, will be discharged up to $50 \%$ DOD only with the integration of the new BVR. The increase in cycle life of the BB due to this is 3000 cycles from 2250 cycles [5].

In a critically designed $\mathrm{PV}$ power source, the BB is designed to take care of three no-sunshine days, and the PA takes two days to replenish the energy.

From assumption (3), it can be concluded that the time interval between two consecutive deep discharges is five days. Therefore, the life of the BB will be given as

with conventional BVR-11250 d

with new BVR-15000 d

The failure rate of the BB wll be calculated from

$$
\begin{aligned}
& f=10^{9} h \backslash M T B F \\
& \text { with conventional BVR-3703 } \\
& \text { with new BVR }-2278
\end{aligned}
$$

The failure rate of the BVR will be calculated by performing an arithmetic summation of the failure rates of the components [6] constituting it. The components with their count and failure rate are given in Tables 1 and 2 for the conventional BVR and the new BVR, respectively.

From the arithmetic summation, the failure rate of the conventional BVR is 16000 . Similarly, 
Table 2. Calculation of failure rate of new BVR based on standard reliability level electronic parts

\begin{tabular}{|c|c|c|c|c|}
\hline \multirow[b]{2}{*}{ S. No. } & \multirow[b]{2}{*}{ Components } & \multirow{2}{*}{$\begin{array}{c}\text { Quantity/unit } \\
(n)\end{array}$} & \multicolumn{2}{|c|}{$\begin{array}{l}\text { Failure rate } \\
\left(\text { per } 10^{9} \mathrm{~h}\right)\end{array}$} \\
\hline & & & $(f)$ & $n \times f$ \\
\hline 01. & Resistance (fixed) & 66 & 58 & 3528 \\
\hline 02. & Variable resistances & 07 & 122 & 854 \\
\hline 03. & Integrated circuits & 08 & 168 & 1344 \\
\hline 04. & Transistor & 10 & 250 & 2500 \\
\hline 05 . & Diode & 15 & 84 & 1260 \\
\hline 06. & Electrolytic capacitor & 10 & 429 & 4290 \\
\hline 07. & Ceramic capacitor & 14 & 214 & 2996 \\
\hline 08. & Switch & 01 & 200 & 200 \\
\hline 09. & Terminal connector & 06 & 100 & 600 \\
\hline 10. & Solder point (mech.) & 355 & 04 & 1420 \\
\hline \multirow[t]{2}{*}{11.} & MOSFET & 03 & 40 & 120 \\
\hline & & & & 19412 \\
\hline
\end{tabular}

the failure rate of the new BVR is 19419. Now, the failure rate of the PV system can be calculated for both BVR. The failure rate with the conventional BVR is

$$
f_{\mathrm{BB}}+f_{\mathrm{BVR}}=3703+16000=19703
$$

The failure rate with the new BVR is

$$
\mathrm{f}_{\mathrm{BB}}+f_{\mathrm{BVR}}=2777+19412=22189
$$

As already mentioned, failure rates increase with an increase in either temperature or humidity or both. Since both conditions vary widely during a whole day and night as well as with seasonal variation, it is very difficult actually to calculate the effects of these. For simplification, the mean ambient temperature will be considered to be $35^{\circ} \mathrm{C}(308 \mathrm{~K})$, and the mean relative humidity will be considered to be $85 \%$ for tropical countries like India. So, by substituting $T=308 \mathrm{~K}, T_{0}=298 \mathrm{~K}$ $\left(25^{\circ} \mathrm{C}\right)$ and $E a=0.8 \mathrm{eV}$ in equation (19), the failure rate becomes $f=2.748 f_{0}$.

Similarly, replacing $R H_{1}=80 \%$ and $R H_{0}=65 \%$ in equation (20), the rate of failure increases by

$$
f_{1}=f_{0}\left(R H_{1} \backslash R H_{0}\right)^{n}=(0.85 \backslash 0.65)^{2.7}=2.06
$$

where $65 \% R H$ and $25^{\circ} \mathrm{C}$ is the base condition for failure rate. Taking into account the cumulative effect of temperature as well as humidity, the failure rate increases by the factor $F$, given by

$$
F=2.06 \times 2.748=5.66
$$

and the failure rate increases for the two BVRs are given as

with conventional BVR-111518

with new BVR-125589

Table 3. Probability of success of various components of a PV system

\begin{tabular}{llcc}
\hline & \multicolumn{1}{c}{ Components/system considered } & $P(s)=\exp [-(f t)] \times 100(\%)$ \\
\hline S. No. & $t=5 \mathrm{yr}$ & $t=10 \mathrm{yr}$ \\
\hline 01. & BB with conventional BVR & 85.03 & 72.3 \\
02. & BB with new BVR & 88.54 & 78.40 \\
03. & Conventional BVR & 49.61 & 24.62 \\
$04 . \quad$ New BVR & 42.73 & 18.26 \\
$05 . \quad$ Rest of the system with conventional BVR & 42.19 & 17.80 \\
06. & Rest of the system with new BVR & 37.84 & 14.32 \\
07. & Rest of the system with conventional BVR at mean temp. $35^{\circ} \mathrm{C}$ and $R H 85 \%$ & 0.756 & 0.057 \\
08. & Rest of the system with new BVR at mean temp. $35^{\circ} \mathrm{C}$ and $R H 85 \%$ & 0.41 & 0.02 \\
\hline
\end{tabular}


The confidence level in the system is given in terms of the probability of success $P(s)$ of the items/system. This is tabulated in Table 3 for periods of 5 and $10 \mathrm{yr}$.

From Table 3, it can be seen that, although the confidence level, i.e. $P(s)$, of the BB increases with the incorporation of the new BVR in the system, the $P(s)$ of the BVR decreases. It is because the improvements in performance are achieved by incorporating additional electronic control blocks. Each block, in turn, adds additional components and, thus, contributes to a higher failure rate. Thus, the gain achieved in the $P(s)$ of the BB is lost in the $P(s)$ of the BVR. Also, the $P(s)$ of the system decreases because the lion's share of the failure rate is contributed by the BVR. From Table 3, it can also be observed that the confidence level decreases to a very low value of less than $1 \%$ for a place with a mean temperature of $35^{\circ} \mathrm{C}$ and mean relative humidity of $85 \%$. That means that, if a hundred systems are deployed in the field, none of them will survive after $5 \mathrm{yr}$ in tropical countries like India. This illustrates the very poor picture of the system performance.

\section{METHODS FOR IMPROVEMENT OF RELIABILITY}

In this section, we will deal with the different reliability engineering techniques to improve upon the reliability estimate obtained above.

The most important criteria for increasing the reliability are as follows:

(1) reduce the complexity of the system to the minimum essential components required for the operation (the non-essential components and unnecessarily complex configurations only increase the probability of system failure);

(2) increase the reliability of the components in the system;

(3) parallel redundancy-one or more (hot) spare components operate in parallel (if one fails, the other still functions);

(4) stand-by redundancy-a (cold) spare part is switched in to take over the function of the component or system that has failed; and

(5) repair maintenance and preventive maintenance.

Application of the above-mentioned techniques is carried out both on the BVR as well as the BB of the PV system.

\section{Criteria applied to $B V R$}

Now, considering the first criterion, the BVR circuit is critically examined, and the following changes are recommended.

(1) Six variable resistances are used in the new BVR. In addition to increasing the number of components in the circuitry, it also adds uncertainty in system performance due to the figuring of variable resistances in the field. So, these can be eliminated by fixed settings of parameters in the laboratory or factory.

(2) The current compensation circuit is not required, since the pulse width modulated charging technique is employed. Therefore, the components associated with that circuit are eliminated.

(3) Two gates of IC CD4070 are unused, so one of them can be used to replace two resistances and one transistor.

These analyses result in the deletion of six variable resistances, eight fixed resistances and one transistor. Because of these, the number of solder joints also decreases and the failure rate decreases to 115605 .

Further, the system reliability can be increased if the components used in the system were used with known methods given as screening and derating.

Screening. Only those components are used in the BVR that pass the burn-in test. They are available in the professional market and are known as "high reliability" level electronic parts. Therefore, these components have low failure rates. Major sources of the failure rate are contributed in the circuit by the resistances, transistors, diodes and electrolytic capacitors. At the same time, the cost implication is marginal if they are replaced by their high reliability counterparts. The failure rate of the BVR is calculated and given in Table 4 based on high reliability level 
Table 4. Calculation of failure rate of the new BVR based on "high reliability" level electronic parts

\begin{tabular}{llrrr} 
& & & \multicolumn{2}{c}{ Failure rate } \\
\cline { 4 - 5 } S. No. & \multicolumn{1}{c}{ Components } & $(n)$ & $(f)$ & $n \times f$ \\
\hline 01. & Resistance (fixed) & 50 & 16 & 800 \\
02. & Integrated circuits & 08 & 168 & 1344 \\
03. & Transistor & 09 & 49 & 441 \\
04. & Diode & 15 & 33 & 495 \\
05. & Electrolytic capacitor (tantalum) & 10 & 43 & 430 \\
06. & Ceramic capacitor & 14 & 86 & 1276 \\
07. & Switch & 01 & 17 & 17 \\
08. & Terminal connector & 06 & 100 & 600 \\
09. & Solder points & 325 & 04 & 1300 \\
10. & MOSFET & 03 & 40 & 120 \\
\hline & & & & 6823 \\
\hline
\end{tabular}

electronics components [6]. The failure rate of the new BVR at $35^{\circ} \mathrm{C}$ mean temperature and $85 \%$ $R H$ is found to be

$$
f=6823 \times 5.66=38618
$$

The failure rate of the new BVR decreases significantly, but it is still high.

Derating. When a population of devices is operated under the rated conditions, a certain failure rate is observed. This is known as the nominal failure rate. This is due to the operational stresses. If the stresses increase above the rated level, the failure rate increases above the nominal failure rate. Similarly, the observed failure rate decreases when the stresses are decreased below the rated level. In practice, the method of using components of the devices at stress levels below their rated stress level is known as derating. In general, the life of components follow either the "Fifth Power Law" [1] or the Arrhenius principle described earlier. The Fifth Power Law states that the life of components is approximately inversely proportional to the fifth power of the stresses. An electrical device following this "law", if operated at 1.2 times its rated voltage, would have its life reduced to approximately one third the life at rated voltage. The same component, operated at $50 \%$ of rating, would have its life increased by a factor of about 30 over the rated life. As a rule of thumb, if all the components are derated by $50 \%$, or, in other words, the components are selected with voltage, power and current ratings twice the required levels, the Fifth Power Law predicts an increase in life by a factor of 30 for components like resistances, capacitors, switch and terminal points. Similarly, for semiconductors, i.e. diodes, transistors, IC's MOSFET, etc., the life depends upon the junction temperature. For a particular power level, the rise in junction temperature is $100^{\circ} \mathrm{C}$ over the ambient temperature of $25^{\circ} \mathrm{C}$. Similarly, by reducing the power rating by $50 \%$, the junction temperature is reduced by $50^{\circ} \mathrm{C}$. Therefore, the reduction in failure rate of a semiconductor can be given by equation (19) as

$$
f_{1}=f_{0} \exp -\{(0.8 \times 11600)(1 \backslash 373-1 \backslash 323)\}=47
$$

So, the law predicts both decreases in failure rate by a factor of 30 and 47 at standard conditions, respectively. Similarly, the decrease in the above failure rate at mean temperature and mean relative humidity is given by a factor of 5.65 and 8.30 , respectively. Since the temperature rise of the devices is reduced by approximately $50 \%$, due to the above derating criteria, the failure rate of solder joints will also decrease by at least the Fifth Power Law. Based on these criteria, the failure rate of the new BVR is calculated in Table 4. Now, the failure rate of the new BVR becomes

$$
f_{\mathrm{BVR}}=553+289+230=1072
$$

As we saw by derating, the failure rate is reduced drastically, but there are limits to it, in terms of cost, weight, space and similar considerations. Sometimes it is counterproductive because absorbed moisture is not driven out due to the very low temperature rise. In some cases, it increases the circuit complexities, e.g. paralleling of more bipolar transistors to share the current.

In view of the above fact, we have to look for other alternatives listed in the criteria for reducing the failure rate further. 
Therefore, parallel redundancy is introduced in the circuit. The failure rate of the BVR can be given by equation (18) as

$$
\begin{gathered}
1 \backslash f_{2}=1 \backslash f_{1}+1 \backslash 2 f_{1}=3 \backslash 2 f_{1} \\
f_{2}=2 \backslash 3 f_{1}
\end{gathered}
$$

where $f_{2}$ is the failure rate after redundancy is introduced and $f_{1}$ is the initial failure rate. If stand-by redundancy is introduced, the reliability of the system is the probability that unit one succeeds for the whole period $(t)$ or that unit one fails at some time $t^{\prime}$ prior to $t$ and the sensing and switching unit two does not fail by $t^{\prime}$ and successfully functions for the reminder of the mission.

Assuming a $100 \%$ reliability of the sensing and switching units one and two while idling, the reliability of the system is given as

$$
\begin{aligned}
& R(t)=R_{1}(t)+Q_{1}\left(t^{\prime}\right) R_{2}\left(t-t^{\prime}\right) \\
& R(t)=\exp \left[-\left(f_{1} t\right)\right]+f_{1}\left\{\exp -\left(f_{1} t\right)\right\}\left\{\exp -\left(f\left(t-t^{\prime}\right)\right)\right\} \\
& R(t)=\exp [-(f t)]+f \exp [-(f t)]
\end{aligned}
$$

The MTTF for a two-unit stand-by system can be determined by equation (7)

$$
\begin{aligned}
\operatorname{MTTF}=R(t) \mathrm{d} t= & \exp -(f t) \mathrm{d} t+f(t) \exp -(f t) \mathrm{d} t \\
& =1 \bigvee f+f f^{2}
\end{aligned}
$$

By comparing the results of equations (23) and (27), it can be concluded that stand-by redundancy is superior to parallel redundancy.

Therefore, if stand-by redundancy is provided, the failure rate of the BVR decreases to

$$
f_{\mathrm{BB}}=1072 / 2=536
$$

This level of failure rate is optimum, but it can be decreased further by the super-reliability technique used in computer hardware, that is stand-by redundancy at the block level instead of at the unit level. This is illustrated in Fig. 2(a) and (b). In configuration (a), any single failure in $\mathbf{M}$ plus any single failure in $\mathbf{M}^{\prime}$, however, will cause system failure. In configuration (b), the system is invulnerable to all single failures and to all double failures except when similar modules fail in $\mathbf{M}$ as well as in $\mathbf{M}^{\prime}$.

From Fig. 1(b), it can be seen that the new BVR is comprised of seven sections. Assuming that the failure rate of each section is equal:

$$
f_{n}=f_{1}=f_{2}=\ldots f_{7}=536 \backslash 7=77
$$

(a)

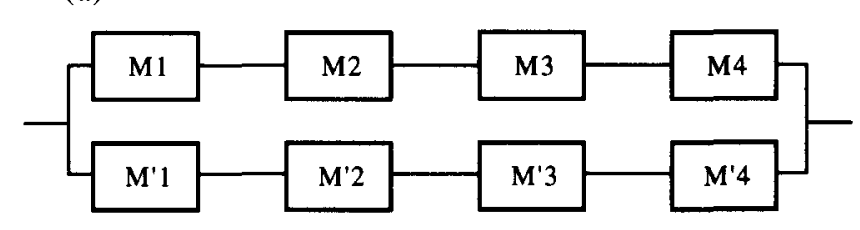

(b)

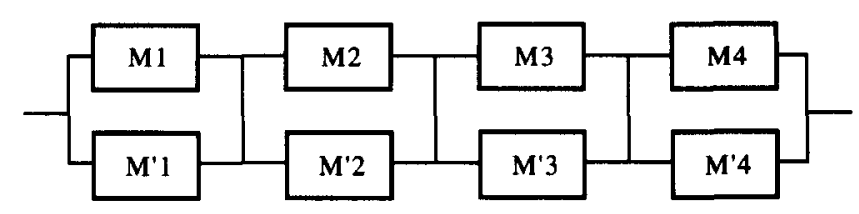

Fig. 2(a). System level redundancy, (b) module level redundancy. 
The module level duplication can be in both ways, i.e. parallel redundancy as well as stand-by redundancy arrangements. The failure rate of module level duplication can be given by equation (18) as

$$
1 \backslash f_{n}^{\prime}=1 \backslash f_{n}+1 \backslash 2 f_{n}=52
$$

If the arrangement is stand-by redundancy, then the failure rate is given by equation (27).

$$
f_{n}^{\prime}=f_{n} \nmid 2=38.5
$$

In this case, the failure rate is quite low.

The first criterion applied to a BVR is not applicable to the BB. The second criterion, i.e. screening and derating, can be applied to the BB also.

(1) Screening. Individual batteries can be screened by charge-discharge cycles. Only those cells that have similar characteristics are used in the BB.

(2) Derating. The BB can be derated in terms of its capacity.

As a rule of thumb, BB capacity is also doubled. As a consequence, the discharge rate is also half; assuming a $10 \%$ rise in capacity due to the decrease in discharge rate [7], the BB will be discharged only up to $30 \%$.

Therefore, the life-cycle of the BB increases to 4200 cycles. Since the capacity of the BB is doubled, it will be discharged in six no-sunshine days and the PV array will take four full-sunshine days to charge it. From assumption (3), it can be concluded that the time interval between consecutive deep discharges is $10 \mathrm{~d}$, so the life of the BB is

$$
4200 \times 10=42000 \mathrm{~d}
$$

and the failure rate $f(\mathrm{BB})$ is

$$
10^{9} \backslash(42000 \times 24)=992
$$

The third criterion can also be applied, but only in very critical systems. The BB cannot be put in a stand-by arrangement, as periodical charging of the BB is necessary.

Cell level redundancy can not be applied because isolating the faulty cell is very difficult.

\section{RESULTS AND DISCUSSION}

Based on the failure rate estimated in the previous section, the confidence level or $P(s)$ can be calculated. This is given in Table 5 for different criteria, one by one, for the BVR.

Similarly, the $P(s)$ for the BB is given as

$$
\begin{array}{rl}
\text { for } t=5 \mathrm{yr} & P(s)=95.75 \% \\
\text { for } t=10 \mathrm{yr} & P(s)=91.68 \%
\end{array}
$$

Table 5. Cumulative effect on probability of success of BVR based on various methods of reliability improvement

\begin{tabular}{llccc}
\hline & & Failure rate & \multicolumn{2}{c}{$P(s)=\exp [-(f t)] \times 100$} \\
\cline { 5 - 6 } S. No. & \multicolumn{1}{c}{ Criteria/method } & (per $\left.10^{9} \mathrm{~h}\right)$ & $t=5 \mathrm{yr}$ & $t=10 \mathrm{yr}$ \\
\hline 01. & Reduction of components & 115605 & 0.06 & 0.04 \\
02. & Screening & 38618 & 18.42 & 3.4 \\
03. & Derating & 1072 & 95.41 & 91.04 \\
04. & Redundancy & 536 & 97.68 & 95.41 \\
05. & Module level parallel redundancy & 52 & 99.77 & 99.54 \\
06. & Module level stand-by redundancy & 38.5 & 99.83 & 99.66 \\
\hline
\end{tabular}


The resultant $P(s)$ of the rest of the system will be derived by first calculating the resultant failure rate

$$
\begin{aligned}
f & =f_{\mathrm{BVR}}+f_{\mathrm{BB}} \\
& =992+38.5=1030.5 \\
P(s) & =91.37 \quad \text { for } 10 \mathrm{yr} \text { period }
\end{aligned}
$$

Reliability levels of more than this are not required because the failure rate of other integral components, e.g. single load/multiple loads, will need maintenance, and faulty sections of the BVR or defective cells can be repaired simultaneously.

Table 5 shows the effect of various techniques on reliability estimates. From the table, the following conclusions can be drawn.

(1) The failure rate drops significantly if components are derated by $50 \%$ and module level duplication is adopted.

(2) Stand-by redundancy is superior to parallel redundancy. At the module level, stand-by redundancy will require high quality change-over switches and sensing circuitry, and the reliability of selection circuits begins to dominate system reliability.

In the case of the BB, its life is increased by derating. As already described, stand-by and module level redundancy cannot be provided in the BB, but parallel redundancy is sometimes provided in very critical systems. In parallel redundancy, one of the BBs is put on the system. So, the total capacity becomes the same. The gain achieved in terms of life cycle due to a decrease in discharge rate is not achieved by providing parallel redundancy because the discharge rate remains the same.

In addition to the above, the system engineer can predict the periodical visits of maintenance people and the cost incurred upon it from the failure rate, while working out the cost of the PV system.

A curve can be drawn between the annual cost of maintenance and the annual cost of reliability as given in Fig. 3, and a trade-off point "a" can be found, to be used in the system design, where the annualised cost of both taken together is at a minimum.

Although reliability depends a lot on design and selection of components, it is equally vulnerable to production processes, etc. Therefore, reliability should be maintained through good quality management [8].

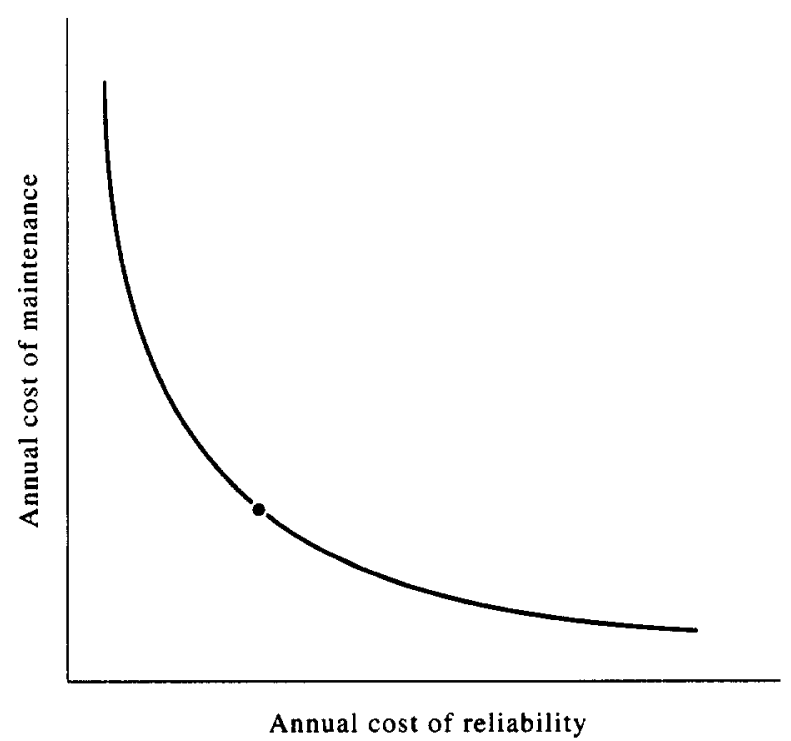

Fig. 3. Curve for the annual cost of maintenance and the annual cost of reliability. 


\section{CONCLUSION}

Reliability estimation for the components of a PV system is very important. Various methods of reliability improvement are available, depending upon the limitations imposed on the system engineer in terms of availability, weight, size and cost of components/items.

From the curve given in Fig. 3, a system engineer can choose different techniques for the least annualised cost of the PV system. The reliability engineering estimation techniques are also useful for the customer to choose a particular item from various similar items offered to him by various suppliers.

\section{REFERENCES}

1. C. O. Smith, Introduction to Reliability in Design. McGraw Hill, New York (1976).

2. E. A. Amrasekra and D. S. Compbell, Failure Mechanism in Semiconductor Devices. Wiley, New York (1987).

3. D. Stewark Peck, Comprehensive model for humidity testing correlation. In IEEE Proceedings of 24th Symposium of Reliability Physics, p. 44 (1986).

4. P. R. Mishra, A. K. Pandey and J. C. Joshi, Sol. Energy Mater. Sol. Cells. 37, 159-174 (1955).

5. S. J. Lancashire, Life-cycle of different battery types for use with photovoltaic systems. IEEE, p. 1157 (1988),

6. MIL-HDBK-217 B, Reliability Prediction of Electronic Equipment (1982 and later revisions), Department of the Navy, Philadelphia, PA.

7. George Wood Vinal, Storage Batteries. Wiley, New York (1955).

8. G. W. A. Dummer and B. Norman, Electronics Reliability Calculation and Design. Pergamon Press, Oxford (1966). 